This item was submitted to Loughborough's Research Repository by the author.

Items in Figshare are protected by copyright, with all rights reserved, unless otherwise indicated.

\title{
The effect of increasing strength and approach velocity on triple jump performance
}

\section{PLEASE CITE THE PUBLISHED VERSION}

http://dx.doi.org/10.1016/j.jbiomech.2016.10.009

\section{PUBLISHER}

(c) Elsevier

\section{VERSION}

AM (Accepted Manuscript)

\section{PUBLISHER STATEMENT}

This work is made available according to the conditions of the Creative Commons Attribution-NonCommercialNoDerivatives 4.0 International (CC BY-NC-ND 4.0) licence. Full details of this licence are available at: https://creativecommons.org/licenses/by-nc-nd/4.0/

\section{LICENCE}

CC BY-NC-ND 4.0

\section{REPOSITORY RECORD}

Allen, Sam, Fred Yeadon, and Mark King. 2016. "The Effect of Increasing Strength and Approach Velocity on Triple Jump Performance”. Loughborough University. https://hdl.handle.net/2134/23027. 
Journal of Biomechanics, in press.

\title{
The effect of increasing strength and approach velocity on triple jump performance
}

\author{
${ }^{1}$ Sam J. Allen, ${ }^{1}$ M.R. (Fred) Yeadon, and ${ }^{1}$ Mark A. King \\ ${ }^{1}$ School of Sport, Exercise, and Health Sciences, Loughborough University, Leicestershire, LE11 3TU, UK
}

\begin{abstract}
The triple jump is an athletic event comprising three phases in which the optimal phase ratio (the proportion of each phase to the total distance jumped) is unknown. This study used a planar whole body torque-driven computer simulation model of the ground contact parts of all three phases of the triple jump to investigate the effect of strength and approach velocity on optimal performance. The strength and approach velocity of the simulation model were each increased by up to $30 \%$ in $10 \%$ increments from baseline data collected from a national standard triple jumper. Increasing strength always resulted in an increased overall jump distance. Increasing approach velocity also typically resulted in an increased overall jump distance but there was a point past which increasing approach velocity without increasing strength did not lead to an increase in overall jump distance. Increasing both strength and approach velocity by $10 \%, 20 \%$, and $30 \%$ led to roughly equivalent increases in overall jump distances. Distances ranged from $14.05 \mathrm{~m}$ with baseline strength and approach velocity, up to $18.49 \mathrm{~m}$ with $30 \%$ increases in both. Optimal phase ratios were either hop-dominated or balanced, and typically became more balanced when the strength of the model was increased by a greater percentage than its approach velocity. The range of triple jump distances that resulted from the optimisation process suggests that strength and approach velocity are of great importance for triple jump performance.
\end{abstract}

Keywords: computer simulation, phase ratio, optimisation, technique

\section{INTRODUCTION}

The triple jump is an athletic event involving three consecutive phases during which athletes must distribute their 'effort' in order to maximise the total distance. Hay (1993) stated that the peak ground reaction forces (GRFs) recorded during the support phase of the step in triple jumping are 'much greater than a human limb is exposed to in any other voluntary activity for which data could be found'. Measured forces range from 12.6 to 22.3 times bodyweight (Amadio, 1985; Ramey and Williams, 1985; Perttunen et al., 2000). Given the magnitude of these peak GRFs it is reasonable to suggest that strength is of great importance to triple jump performance. However, the isolated effects of changes in strength on performance are hard to gauge experimentally. Increasing strength has been shown to improve optimal performance in computer simulations of vertical squat jumping in which the height reached is solely determined by the amount of work done by the muscles (Bobbert and van Soest, 1994). Seyfarth et al., (2000) found that the outcomes of computer simulations of the long jump were particularly sensitive to muscle strength and eccentric force enhancement, but the mechanism for improvement in performance in a running jump is harder to define, since it cannot be easily related to work done by muscles; there is no simple relationship between energy and performance.

During the ground contact of a running jump, horizontal velocity is 'converted' to vertical velocity as the centre of mass (CoM) 'pivots' over the foot; vertical velocity can be generated whilst the joints of the stance leg are flexing (Dapena and Chung, 1988). Horizontal velocity must therefore be 'traded off' against vertical velocity. A comparison between high jumping and long jumping indicates that athletes achieve the higher vertical takeoff velocities needed for high jumping by planting the stance leg at a larger 
angle from the vertical, putting the CoM of the body lower and further behind the foot (Alexander, 1990; Wilson et al., 2011). This causes the angle between the velocity vector of the CoM and the vector from the CoM to the centre of pressure (CoP) (the 'radius' of the circle on which the CoM pivots) to decrease, leading to a higher inwards radial velocity (i.e. the distance between the CoM and the CoP shortens) and a lower tangential velocity (Dapena and Chung, 1988). During this period the joints of the stance leg, especially the knee, are in eccentric conditions and are therefore dissipating energy. Typically, proportionately more horizontal velocity is lost as gains in vertical velocity increase, due to the requisite increase in plant angle leading to more energy dissipation by the stance leg and larger changes in potential energy of the mass centre. It has been proposed that the ability of an athlete to 'convert' horizontal velocity to vertical velocity is subject-specific (Yu and Hay, 1996; Allen et al., 2013) but the effects of strength and approach velocity on this relationship have not been investigated. It is possible that an increase in strength would allow a more efficient conversion of horizontal velocity to vertical velocity because the leg would be better able to resist flexion, and hence energy dissipation due to eccentric muscle actions.

The 'phase ratio' comprises the distances of each phase expressed as three percentages of the total distance. Triple jump techniques have been defined as being: (a) hop-dominated - where the hop percentage is at least $2 \%$ greater than the next largest phase percentage; (b) jump-dominated - where the jump percentage is at least $2 \%$ greater than the next largest phase percentage; and (c) balanced - where the largest phase percentage is less than $2 \%$ greater than the next largest phase percentage (Hay, 1992). There have been a number of attempts to determine the effect of phase ratio on triple jump performance using various approaches including: observations of elite jumpers (Miller and Hay, 1986; Hay, 1992, 1993, 1995, 1997, 1999; Song and Ryu, 2011); the differences between elite and novice jumpers (Simpson et al., 2007); statistical relationships between velocity tradeoffs during the contact phases (Yu and Hay, 1996; Yu, 1999); and even an operations research approach (Brimberg et al., 2006).

Attempts have also been made to optimise technique using a subject-specific computer simulation model of all three phases of the triple jump (Allen et al., 2016). The results indicated that for the individual in the study a hop-dominated or balanced technique would be optimal, and that a jump-dominated technique would lead to a reduction of approximately $3 \%$ in triple jump distance. The best performance of the triple jumper in this study was $14.35 \mathrm{~m}$ which is below that of elite competitors and therefore it is difficult to generalise the findings to an elite population. It has been observed that athletes approach more slowly when triple jumping compared to long jumping, indicating that approach velocity in triple jumping is submaximal (Hay, 1993). Hop-dominated techniques are associated with higher forces than jump-dominated techniques, especially during the step stance phase (Allen et al., 2016); therefore employing a jump-dominated technique may lead to a reduction in GRF magnitude and allow an increase in approach velocity (Hay, 1995), since higher velocities are also associated with higher forces.

In order to generalise technique obtained from a simulation model across a population of athletes of various strengths and sprinting speeds it is necessary to vary these factors during the optimisation process. The aim of this study was therefore to determine the effects of increasing the strength and approach velocity of an athlete on total jump distance and phase ratio using a planar whole body forward dynamics computer simulation model of the ground contact parts of all three phases of the triple jump. In order to fufil this aim, the following specific questions will be answered: 
1) Do increases in strength and approach velocity result in increases in jump distance?

2) Do increases in strength and approach velocity result in altered optimal phase ratios?

3) Do increases in strength and approach velocity change the capacity of the model to convert horizontal to vertical velocity?

4) Do increases in strength and approach velocity change the optimal plant angles at the touchdown of each phase?

\section{METHODS}

\subsection{Data collection and parameter determination}

The study was conducted in accordance with the Loughborough University Ethics Committee guidelines. Subject-specific torque and inertia parameters were calculated from measurements taken from a national standard male triple jumper (age: 22 years; mass: $72.6 \mathrm{~kg}$; height: $1.82 \mathrm{~m}$; best performance: $14.35 \mathrm{~m}$ ). Maximal voluntary joint torque data was obtained, assuming bilateral symmetry, using an Isocom isovelocity dynamometer for flexion and extension of the ankle, knee, hip, and shoulder on the right hand side of the body (King et al., 2006). Ninety-five anthropometric measurements were taken along with body mass and used as input to the inertia model of Yeadon (1990) in order to calculate subject-specific segmental inertia parameters which allowed calculation of the whole body CoM location and moment of inertia. Kinematic data was collected at the Loughborough University indoor High Performance Athletics Centre from a single triple jump performance from an approach run of self-selected length. Forty-five $25 \mathrm{~mm}$ retroreflective markers were placed on the athlete in order that locations of joint centres could be determined. Eighteen Vicon MX cameras, covering a volume of $18 \mathrm{~m} \times 2 \mathrm{~m} \times 2.5 \mathrm{~m}$ spanning the last stride of the approach and the complete triple jump, captured data at $240 \mathrm{~Hz}$. Approach velocity was defined as the horizontal velocity of the whole body CoM at the touchdown of the hop stance phase. The performance resulted in an approach velocity of $8.1 \mathrm{~m} . \mathrm{s}^{-1}$ and a triple jump distance of $13.00 \mathrm{~m}$, employing a balanced technique (35.5\%:30.4\%:34.1\%). Orientation, defined as the angle of the trunk in a global reference frame, and configuration angles were calculated by considering the joint centre coordinates in the sagittal plane.

\subsection{Simulation model}

A 13-segment planar torque-driven computer simulation model was used to investigate triple jumping technique (Allen et al., 2010; Allen et al., 2012). The equations of motion for the system were developed using Autolev ${ }^{\mathrm{TM}}$ which generated code in Fortran using Kane's method. Simulations were integrated over time using a $4^{\text {th }}$ order variable time step Runge-Kutta algorithm with a main time step of $0.0001 \mathrm{~s}$ and absolute and relative error tolerances of $10^{-7}$ and $10^{-8}$ respectively. The 13 segments comprised: head + trunk, two upper arms, two forearms and hands, two thighs, two shanks, two 2-segment feet, with wobbling masses within the shanks, thighs, and torso. The model was driven by torque generators consisting of contractile components and series elastic components which were employed to flex and extend the shoulder, hip, knee, ankle, and ball joints. The elbow joints were angle-driven since it was assumed they would behave similarly to recorded performances. In addition to active torque generators, the ankle, knee, and hip had passive elements (Riener and Edrich, 1999) which produced restorative torques at the extremes of range, acting to stop the limb exceeding anatomical limits. Non-linear spring-dampers connected the ends of the wobbling and fixed elements (Pain and Challis, 2001). Each foot had three points of contact with the ground at the heel, ball (metatarsophalangeal joint), and toe. The foot- 
ground interface was modelled using horizontal and vertical non-linear spring-dampers situated at the heel, ball, and toe of each foot (Allen et al., 2012). Viscoelastic parameters representing the wobbling masses and the foot-ground interface were previously determined via optimisation; these parameters were varied in order to minimise the differences between simulated ground reaction force and kinematic data, and equivalent performance data (Allen et al., 2013). The performance data was matched using a simulation of the complete triple jump (Allen et al., 2016) and this was subsequently used as the base simulation for optimisations.

\subsection{Optimisations}

The strength and approach velocity of the model were each increased by: $0 \%$, $10 \%, 20 \%$, and $30 \%$ from the measured values and all combinations of these two parameters were investigated, leading to 16 optimisations in total. The strength of the model was manipulated by increasing the maximum isometric joint torque of both flexors and extensors at all joints. Approach velocities ranged from $8.1 \mathrm{~m} . \mathrm{s}^{-1}$ to 10.5 $\mathrm{m} . \mathrm{s}^{-1}$ (the maximum approach velocity recorded at the 2009 IAAF World Championships in Berlin [German Athletics Federation, 2009]). Optimisation was used to maximise the distance of the whole triple jump in each condition.

\subsubsection{Jump distance optimisation}

A Genetic Algorithm (GA) (Carroll, 1996) was used to maximise total jump distance by varying 243 parameters: 231 torque generator activation parameters (77 in each phase comprising ramp start times, ramp durations, and activation levels as described by Allen et al. [2010]); and four initial angles in each phase: the orientation angle, and the hip, knee, and ankle angles of the stance leg, giving 12 in total. The GA was implemented with the following characteristics: jump and creep mutations; tournament selection; elitism; niching, and single-point crossover. Probabilities of jump and creep mutation were as advised by Carroll (1996). The population size was 1056 and optimisations were considered to have converged upon a solution when the objective function representing the total jump distance had not improved by more than 1 $\mathrm{cm}$ in 1000 generations, this was typically after 10000 generations. Each of the 16 optimisations therefore necessitated approximately 10 million individual simulations. Optimisations were run in parallel on the Hydra High Performance Computing system at Loughborough University (2460-core 64-bit Intel Xeon cluster), with each optimisation requiring approximately 1 year of processor time, and so using 12 processors per optimisation (192 in total) allowed optimisations to be completed within one month. The vertical CoM velocity at the touchdown of the hop stance phase, and configuration angles and angular velocities at the touchdown of each stance phase, were taken from the matched simulation (Allen et al., 2016). The horizontal and vertical CoM position and velocity, and trunk orientation and whole-body angular momentum at the takeoff from each phase were used in order to calculate the linear CoM velocity, and trunk orientation and angular velocity at the touchdown of the subsequent phase (Allen et al., 2016).

In the airborne phases orientation changes were assumed to be the same as those calculated from the measured performance configuration changes. Orientation changes and average whole body moment of inertia were determined by running an angle-driven simulation of each flight phase with no angular momentum. This allowed the initial orientation of each phase to be calculated from the takeoff orientation and whole body angular momentum of the previous phase. The calculated initial orientations were allowed to vary in each phase, since it was assumed that takeoff configurations and airborne motions different from the measured performance could 
lead to altered orientation changes during flight. The initial orientation of the hop phase was allowed to vary between $\pm 10^{\circ}$ from the matched performance, since it was assumed that the athlete could alter his orientation substantially during the approach run. The bounds on the variations in initial orientation angle in the step and jump phases were set to $\pm 20 \%$ of the magnitude of the changes in orientation angle that performance configuration changes produced in the previous flight phase. This led to bounds of $\pm 5^{\circ}$, and $\pm 2^{\circ}$ respectively from the landing orientations of the step and jump phases calculated from the previous phases. The initial ankle, knee, and hip angles were each allowed to vary by up to $\pm 5^{\circ}$ from the matched simulation in each phase and, together with the orientation angle, these made up the 12 angles included in the optimisation process. The measured performance landing orientation during the jump phase was assumed to be optimal and distance penalties were imposed if simulations deviated from this.

The total distance of each optimised simulation was calculated along with the distance of each constituent phase. A least squares polynomial surface was fit to the data as a function of strength and approach velocity, which were expressed as percentage increases from the measured data, in order to establish the effect of increasing each parameter on jump distance.

\section{RESULTS}

\subsection{Jump distance optimisation}

Optimisation of jump distance with measured strength and approach velocity resulted in a total distance of $14.05 \mathrm{~m}$ and a hop-dominated technique. Increases in strength resulted in increases in jump distances in every condition, whereas increases in velocity led to increases in jump distances in all cases except one (Table 1). Figure 1 gives a visual representation of the techniques employed in selected optimisations.
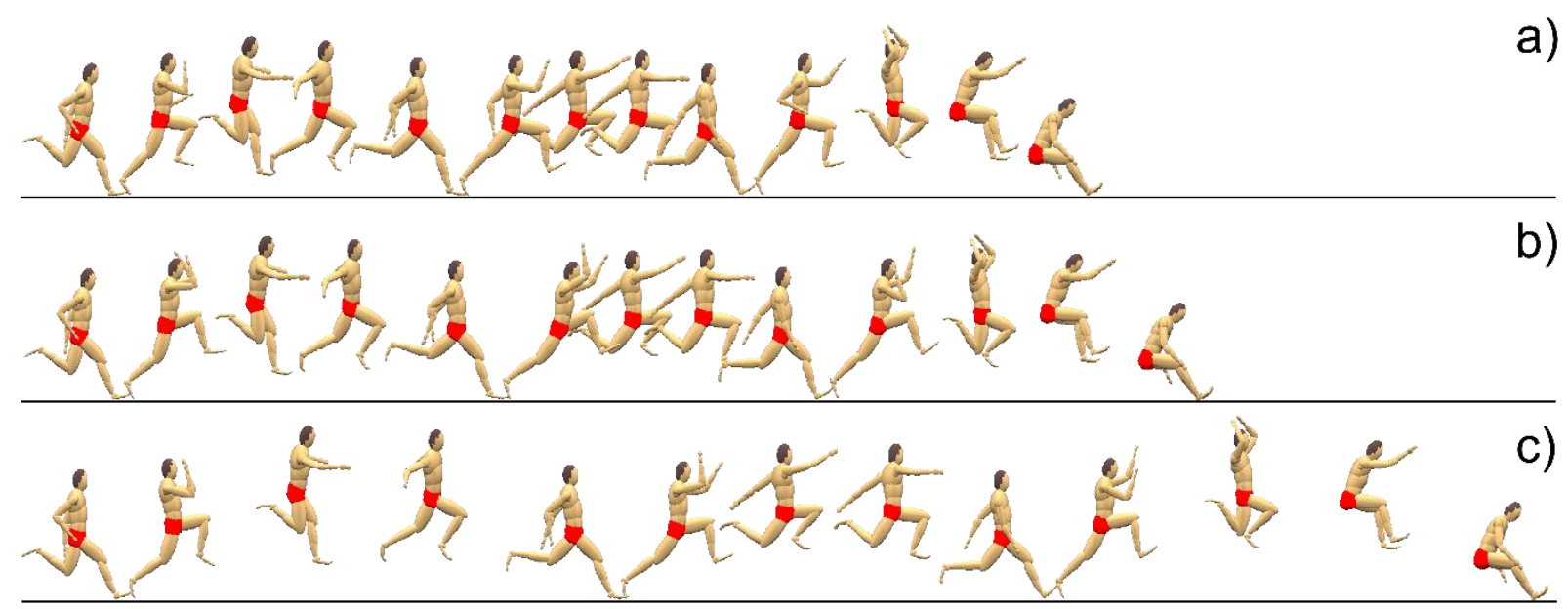

Figure 1. Techniques employed in a) the matched simulation, b) the optimised simulation with $100 \%$ strength and approach velocity, and c) the optimised simulation with $130 \%$ strength and approach velocity. 
Table 1. Overall jump distances

\begin{tabular}{ccccc}
\hline strength & \multicolumn{4}{c}{ velocity } \\
& $\mathbf{1 0 0} \%$ & $\mathbf{1 1 0} \%$ & $\mathbf{1 2 0} \%$ & $\mathbf{1 3 0} \%$ \\
$\mathbf{1 0 0 \%}$ & $14.05 \mathrm{~m}$ & $14.67 \mathrm{~m}$ & $15.12 \mathrm{~m}$ & $15.12 \mathrm{~m}$ \\
$\mathbf{1 1 0} \%$ & $14.87 \mathrm{~m}$ & $15.54 \mathrm{~m}$ & $16.03 \mathrm{~m}$ & $16.53 \mathrm{~m}$ \\
$\mathbf{1 2 0} \%$ & $15.48 \mathrm{~m}$ & $16.34 \mathrm{~m}$ & $17.10 \mathrm{~m}$ & $17.58 \mathrm{~m}$ \\
$\mathbf{1 3 0} \%$ & $16.20 \mathrm{~m}$ & $17.06 \mathrm{~m}$ & $17.94 \mathrm{~m}$ & $18.49 \mathrm{~m}$ \\
\hline
\end{tabular}

The least squares polynomial fit to the data in Table 1 resulted in an $R^{2}$ value of 0.9974 (Figure 2):

$D=14.11+0.08294 \cdot S+0.06781 \cdot V+0.001404 \cdot S \cdot V-0.000475 \cdot S^{2}-0.0009625 \cdot V^{2}$,

where $D$ is the jump distance, $S$ is strength, and $V$ is approach velocity.

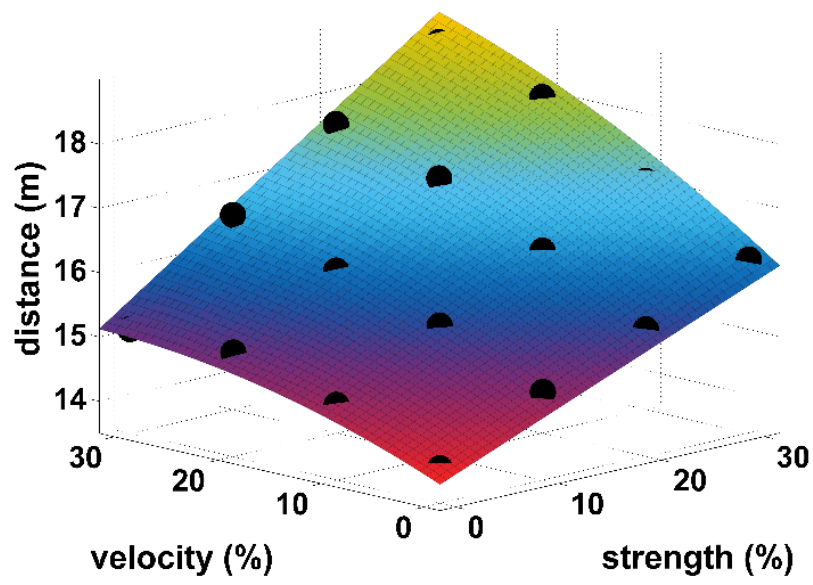

Figure 2. Surface fitted to optimised jump distances (black dots) plotted against increases in velocity and strength.

The phase ratios employed by the model were sensitive to both strength and approach velocity (Table 2). All techniques were either balanced or hop-dominated.

Table 2. Phase ratios

\begin{tabular}{ccccc}
\hline strength & \multicolumn{3}{c}{ velocity } \\
& $100 \%$ & $110 \%$ & $120 \%$ & $130 \%$ \\
$100 \%$ & $35.6 \%: 30.8 \%: 33.6 \%$ & $36.0 \%: 30.4 \%: 33.6 \%$ & $36.6 \%: 28.6 \%: 34.7 \%$ & $35.5 \%: 28.9 \%: 35.5 \%$ \\
$110 \%$ & $34.5 \%: 32.7 \%: 32.8 \%$ & $36.7 \%: 30.1 \%: 33.2 \%$ & $34.6 \%: 31.3 \%: 34.2 \%$ & $36.6 \%: 28.6 \%: 34.7 \%$ \\
$120 \%$ & $34.3 \%: 32.6 \%: 33.1 \%$ & $36.7 \%: 30.5 \%: 32.7 \%$ & $36.2 \%: 30.3 \%: 33.6 \%$ & $36.5 \%: 29.5 \%: 34.0 \%$ \\
$130 \%$ & $34.7 \%: 33.0 \%: 32.3 \%$ & $33.3 \%: 33.3 \%: 33.4 \%$ & $36.4 \%: 31.0 \%: 32.6 \%$ & $35.3 \%: 31.2 \%: 33.5 \%$ \\
\hline
\end{tabular}


Horizontal takeoff velocities were similar throughout the triple jump for a given approach velocity regardless of strength, whereas vertical takeoff velocities increased with increasing strength (Figure 3).

a)

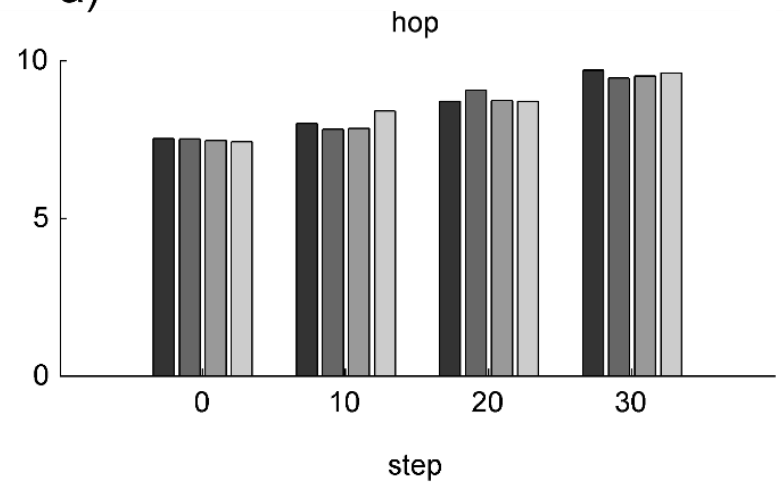

b)

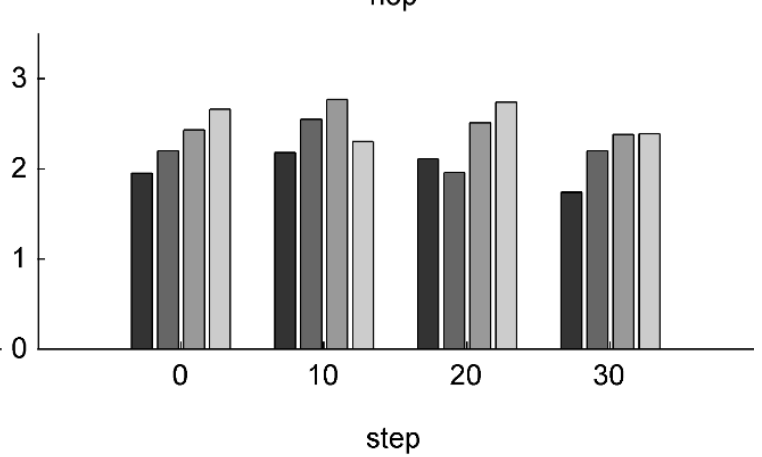

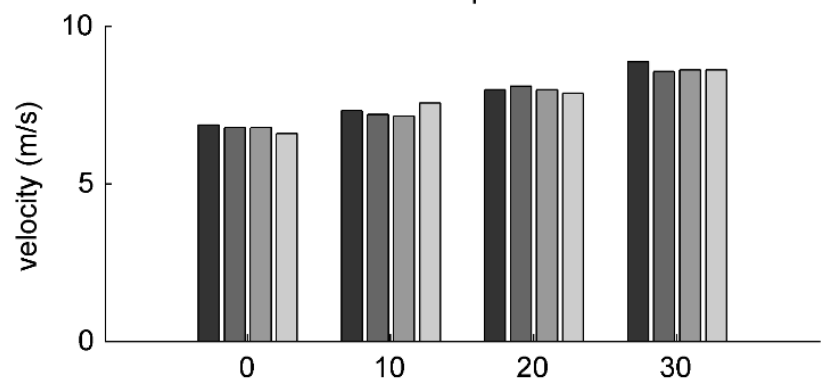
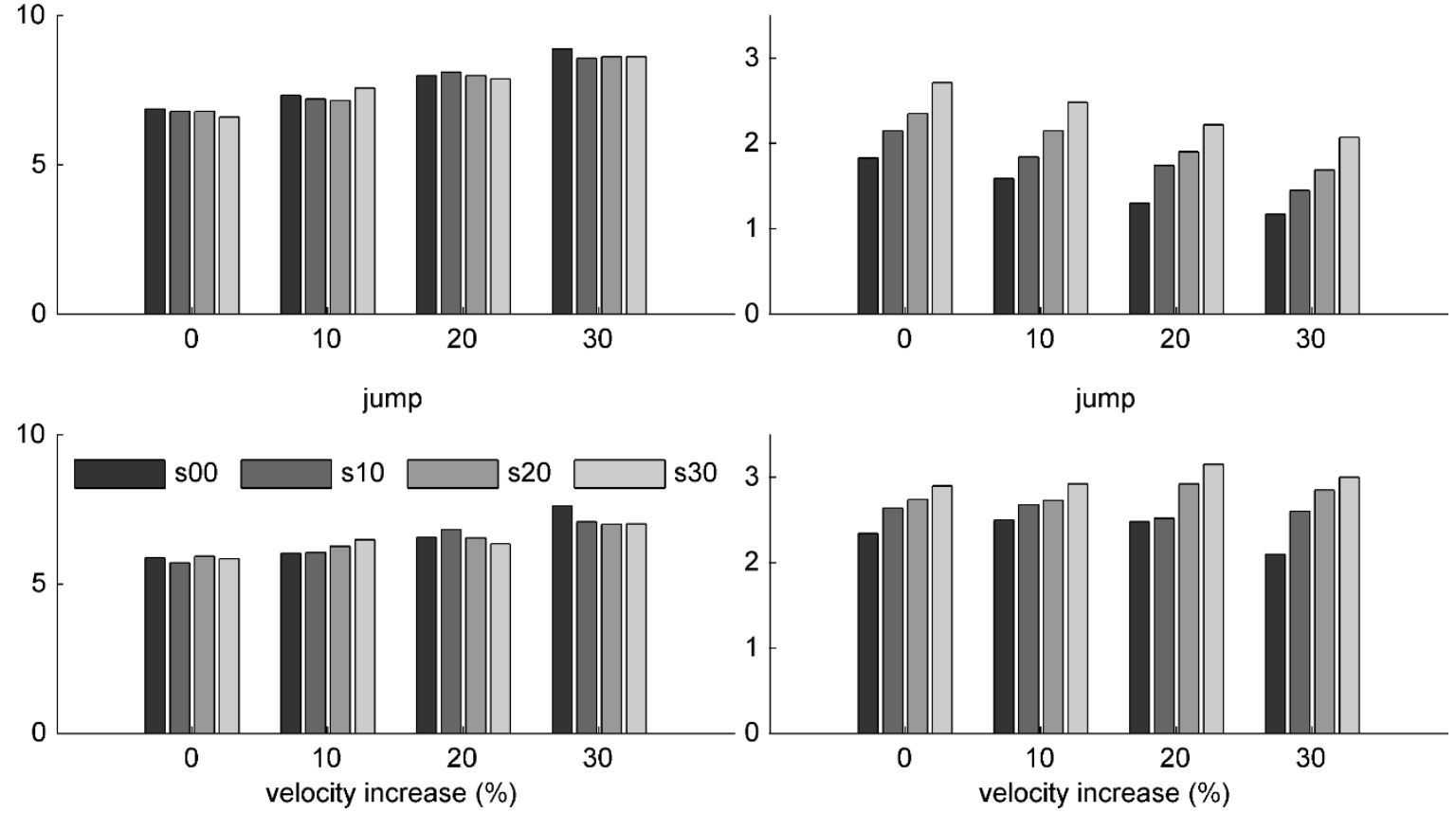

Figure 3. Horizontal a) and vertical b) takeoff velocities for each phase as a function of approach velocity increases (key: s indicates strength, and the following number the increase from baseline). 
Plant angles typically increased with increasing approach velocity, and decreased with increasing strength in the step and jump phases (Figure 4).

a)
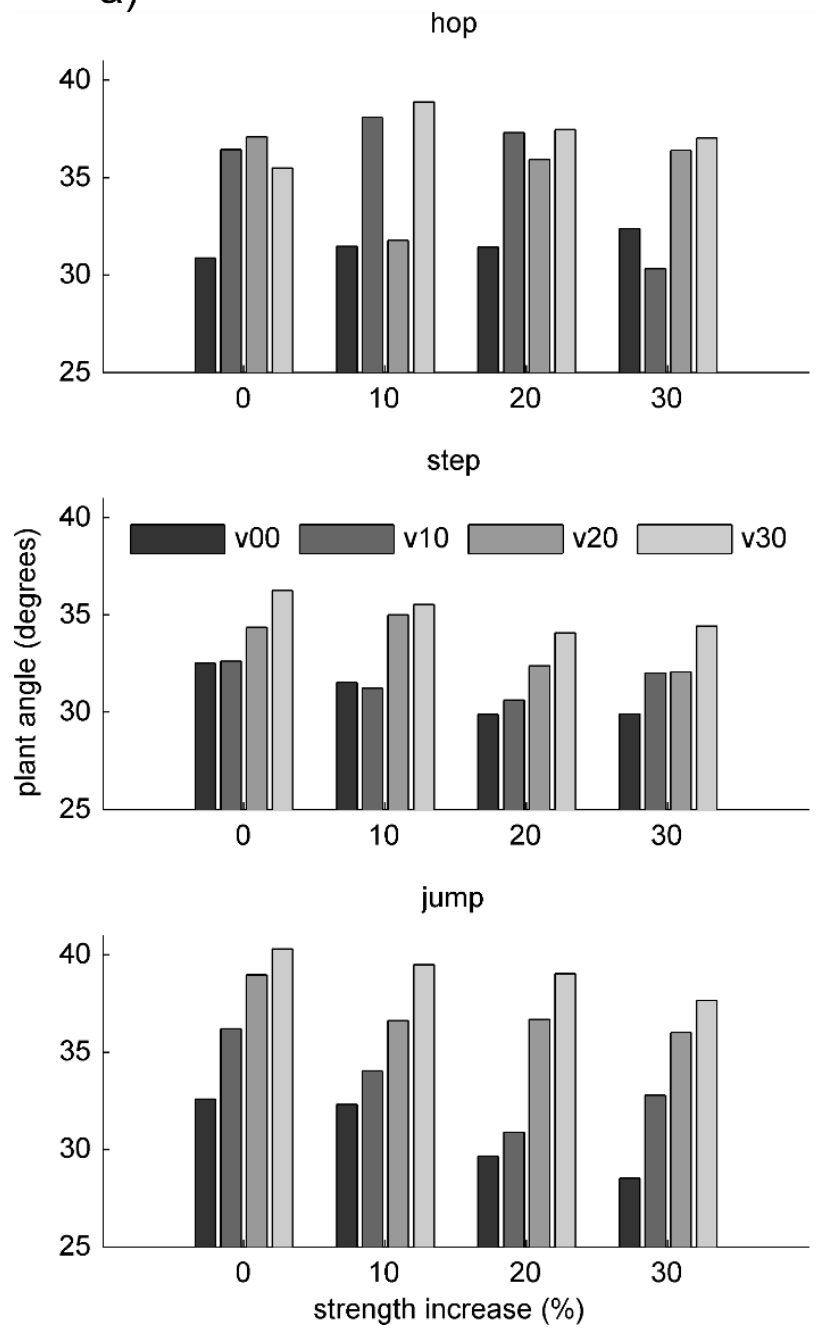

b)
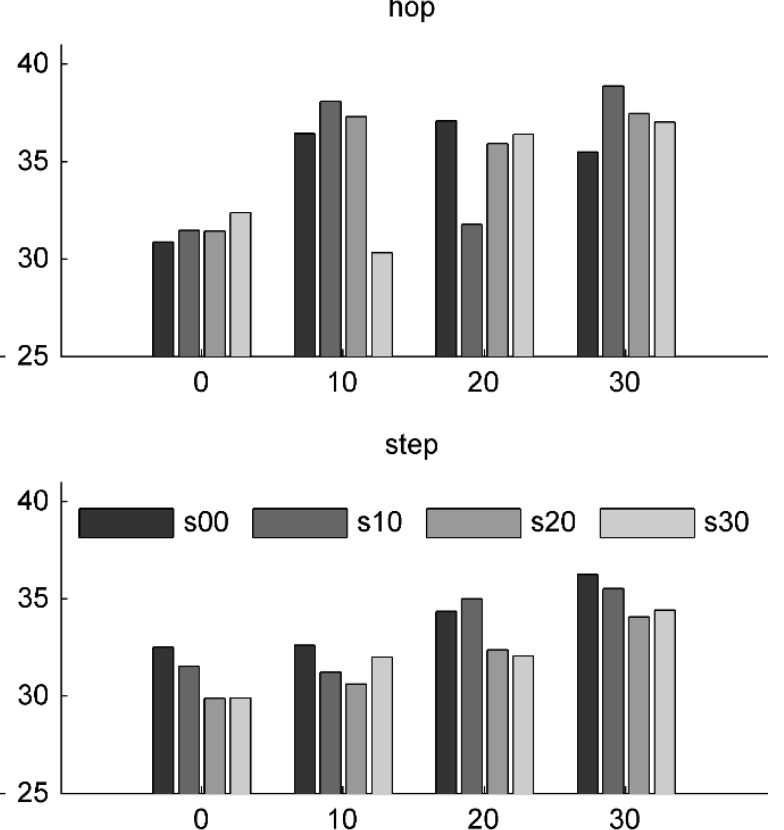

jump

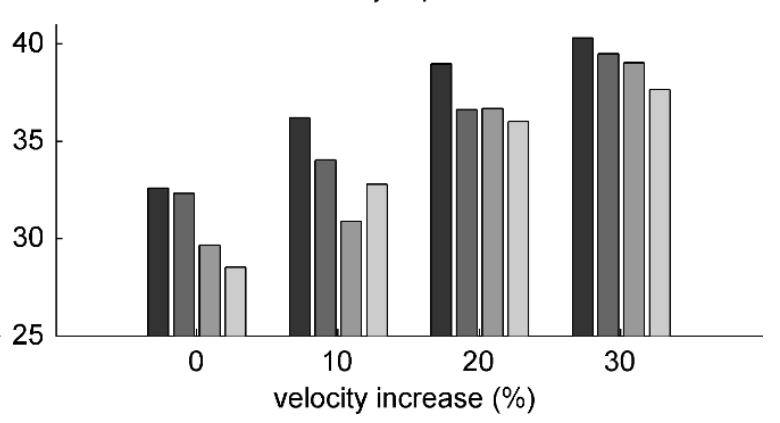

Figure 4. Plant angles as a function of a) approach velocity increases, and b) strength increases (key: $s$ indicates strength, $v$ velocity, and the following number the increase from baseline).

\section{DISCUSSION}

The optimisation results indicated that triple jump distance was sensitive to both strength and approach velocity. Increasing both strength and approach velocity by $30 \%$ led to an overall triple jump distance increase of $31.6 \%(18.49 \mathrm{~m})$, which was in excess of the current men's world record $(18.29 \mathrm{~m})$. In answer to Question 1, increases in strength always resulted in improvements in performance however, there was a point past which increasing approach velocity at the minimum strength level did not result in improved performance (Table 1 ). It is very unlikely that this critical velocity would ever be reached in practice at higher strength levels, since the highest velocity was already at the levels of elite jumpers $\left(\sim 10.5 \mathrm{~m} \cdot \mathrm{s}^{-1}\right)$ and distance was still increasing for increasing velocity. This result indicates that approaching as quickly as possible may be optimal for athletes with high strength levels, assuming they can coordinate the movement at these higher velocities. Triple jumpers do typically approach more slowly than long jumpers and, if this is due to submaximal effort, the findings of this study indicate that this disparity is likely to be a coordination issue, since increasing approach velocity should theoretically always be beneficial to a relatively strong athlete, assuming optimal technique. Equation 1 indicates that a unit improvement in strength was always 
more beneficial than a unit improvement in velocity. However, there was also an interrelationship between strength and approach velocity; increasing strength independently by up to $10 \%$ was most beneficial, after which it became more beneficial to increase both strength and approach velocity.

In answer to Question 2, phase ratios were largely governed by the step phase percentage, which was in turn related to strength and approach velocity. When strength increased with no increase in approach velocity the step phase percentage tended to increase, when approach velocity increased with no increase in strength the step phase percentage tended to decrease, and when strength and approach velocity increased in tandem the step phase percentage remained approximately constant (Table 2). When the step phase was approximately $30-31 \%$ the phase ratio was typically hop-dominated; if it deviated in either direction the jump became balanced. No jump-dominated techniques were found to be optimal, indeed in no optimal simulation was the jump phase the longest phase. This could have been due in part to the fact that the landing configuration of the model was invariant; it is possible that a jump-dominated technique would allow an altered landing configuration (Figure 1). However, even if this were the case then it is unlikely that any of the optimal techniques found in this study would become jump-dominated. It has been proposed that a jump-dominated technique may allow athletes to approach faster than they would otherwise be capable of (Hay, 1995; Allen et al., 2016). However, the results of this study indicate that, even at the highest velocity and the lowest strength level, the optimal phase ratio was balanced. It is possible that athletes who use a jump-dominated technique do so because it is easier to coordinate than other techniques and therefore enables them to be more consistent.

The horizontal velocity at the takeoff of each phase was similar for a given approach velocity regardless of strength, although the vertical velocities were typically higher as strength increased, leading to an increase in overall jump distance (Figure 3). This indicates that the optimisation procedure chose plant angles which resulted in similar losses of horizontal velocity for increased gains in vertical velocity, rather than reduced losses in horizontal velocity for equivalent gains in vertical velocity (Figure 4). In Appendix A a simple analytical model is described for which the optimal loss of horizontal velocity during a horizontal jump is independent of strength. In answer to Question 3, the results of this study indicate that with increasing strength the model could generate higher vertical velocities for similar losses in horizontal velocity, and therefore jump further. An example is shown in Figure 5 where vertical forces, and therefore impulses, increased as strength increased, whereas horizontal forces were similar. It has been proposed that the ability of an athlete to 'convert' horizontal velocity to vertical velocity is subject-specific (Yu and Hay, 1996; Allen et al., 2013). The results of this study indicate that, rather than being entirely subject-specific, it may be dependent on both the strength and approach velocity of the athlete: stronger athletes can achieve more vertical velocity for a unit loss in horizontal velocity, and athletes of a given strength can achieve more vertical velocity for a unit loss in horizontal velocity with decreasing approach velocity.

In answer to Question 4, with increasing approach velocity there was a trend towards larger plant angles in the step and jump phases (Figure 4), this led to increased energy losses due to greater losses in horizontal velocity in favour of generating vertical velocity. With increasing strength there was a less pronounced trend of plant angles decreasing during the step and jump phases. The plant angles required for the model to generate a given vertical velocity decreased with increasing strength, since the leg was better able to resist flexion and hence the shortening of the radial distance between the CoM and CoP; this led to a more efficient transfer of momentum as the CoM did not have to rotate as far about the CoP. This was in agreement with the finding of Seyfarth 
et al. (2000) that long jump performance was particularly sensitive to eccentric force enhancement.

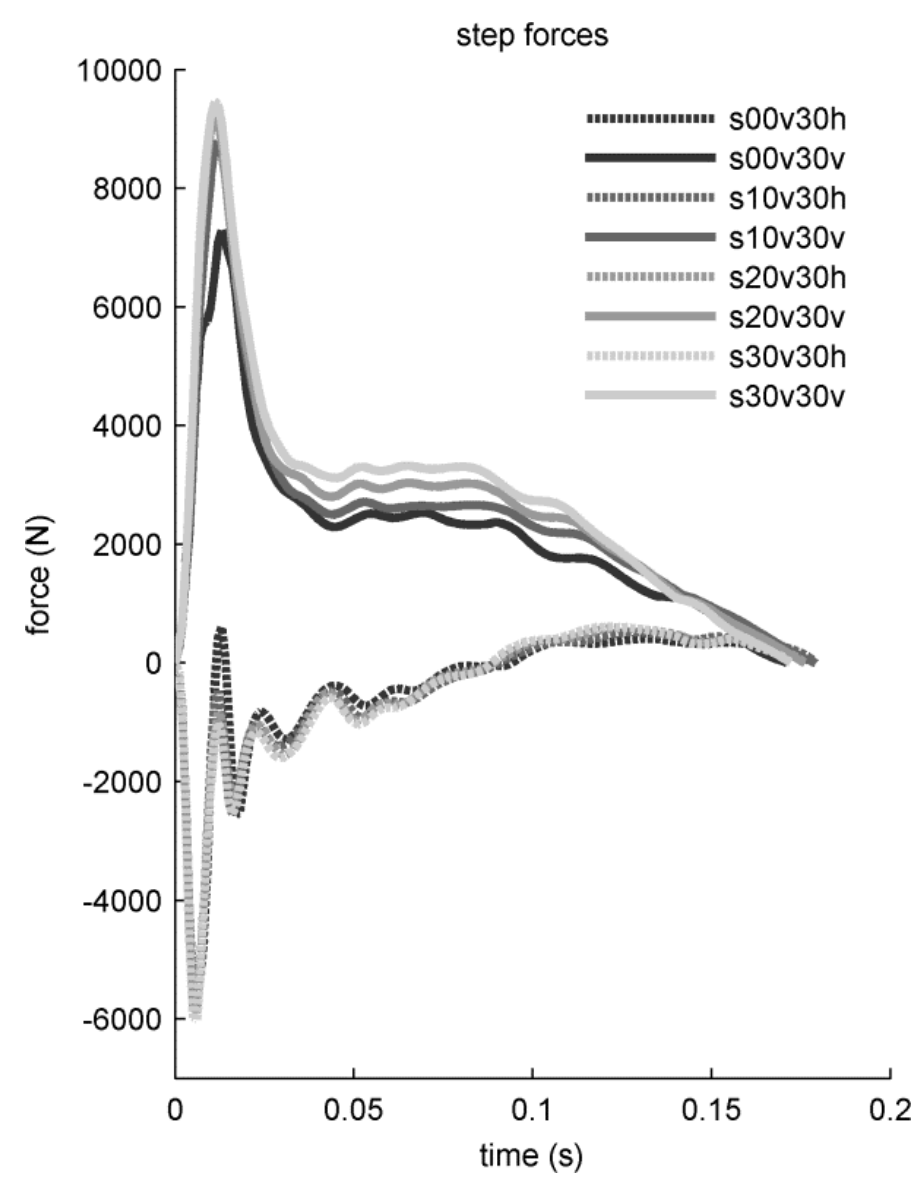

Figure 5. Example horizontal and vertical ground reaction forces (key: $s$ indicates strength, $v$ velocity, and the following number the percentage increase from baseline, $h$ or $v$ appended to the end indicates horizontal or vertical direction).

Torque generator activation timings show that in each case the strategy for the triple jump was largely determined during the hop takeoff, mainly by variation in the knee torque generator activation timings (Figure 6). After this the torque generator activation strategy was broadly the same for the step and jump phases regardless of strength or velocity: ramping up as quickly as possible and ramping down towards the end of the ground contact. This indicates that the hop is the only stance phase that may involve submaximal effort; the step and jump stance phases seem to necessitate applying as much torque as possible, with the kinematic conditions at touchdown determining the energy changes. This may explain why plant angle relationships are only apparent in the step, and jump phases. Altering strength and approach velocity affected the joint torques of the stance leg (Figure 7); increasing strength led to proportionate increases in joint torques, while increasing approach velocity led to similar peak torques achieved sooner since torque generators were forced into high eccentric angular velocities more quickly. With increasing approach velocity angular impulses remained fairly constant at the hip and knee joints, despite briefer ground contacts, and decreased at the ankle. 


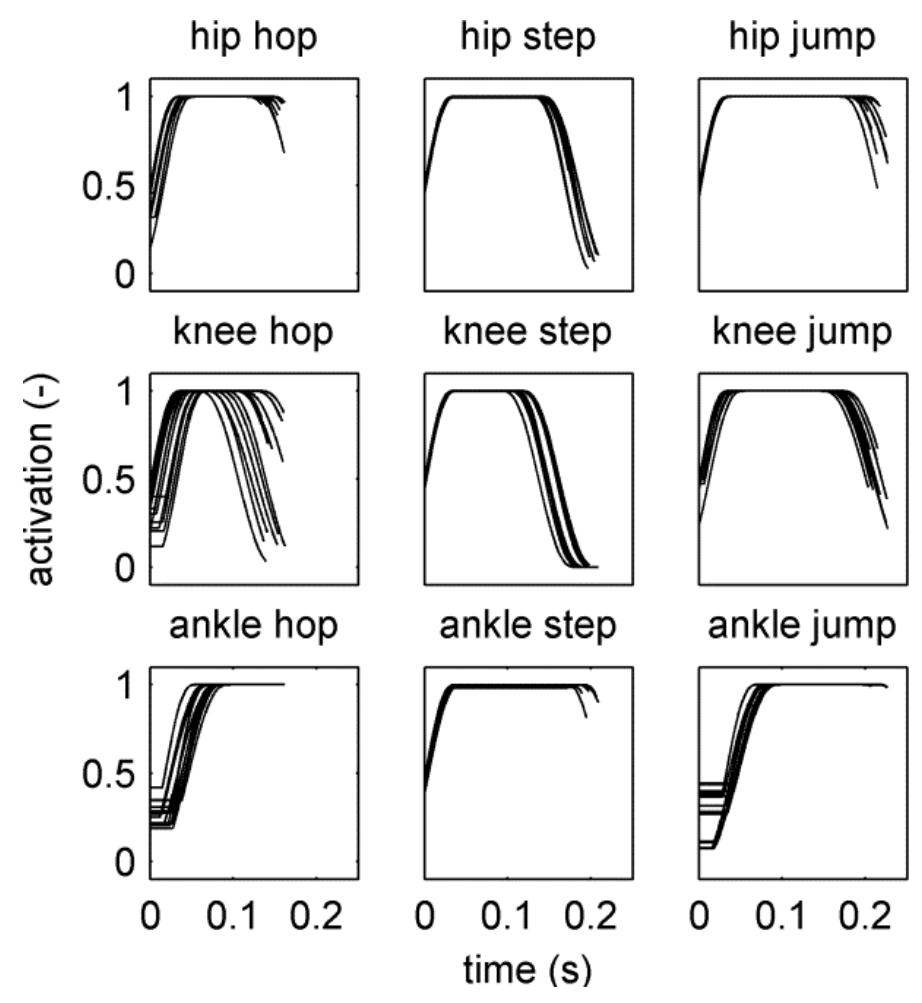

Figure 6. Activation time histories for the joints of the stance leg for all phases and optimisations.

Many aspects of the model employed in this study were subject-specific, these included: segment lengths; inertia parameters; and torque generator parameters (other than maximum isometric torque). This could be considered a limitation when attempting to generalise findings across a population. However, the fact that percentage increases in both strength and approach velocity led to roughly equivalent percentage increases in triple jump distance indicates that these parameters are likely to be very influential. Whilst the aforementioned subject-specific parameters are likely to influence triple jump performance it would seem unlikely that this influence would be of the same order of magnitude as that observed in this study. 
a)

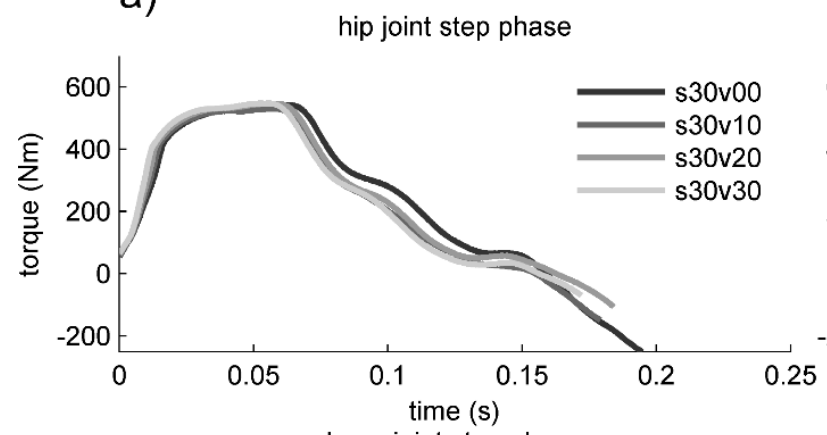

knee joint step phase

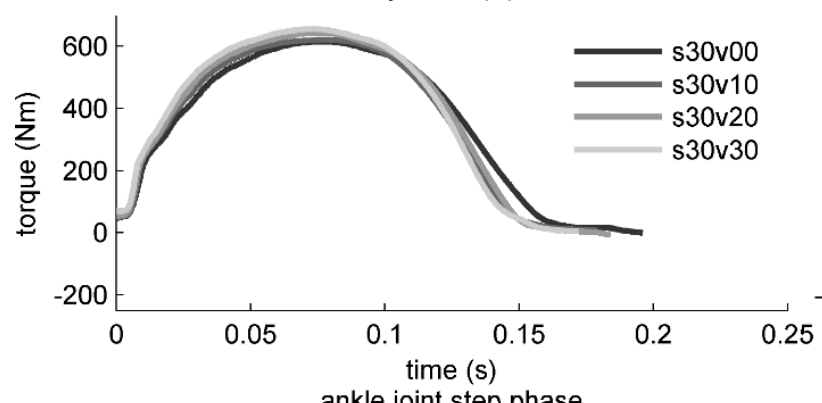

ankle joint step phase

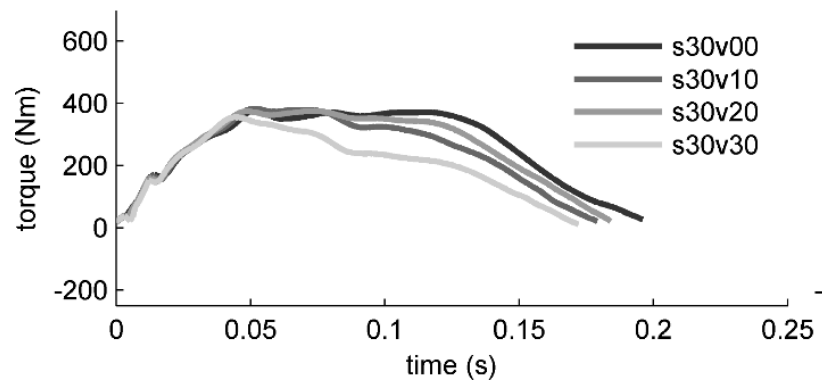

b)

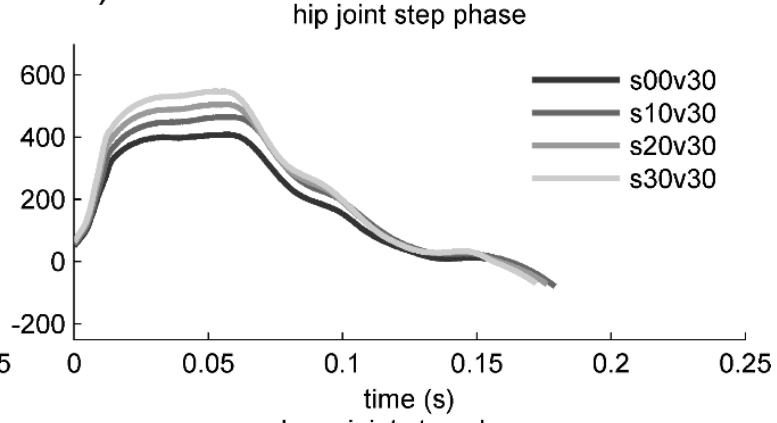

knee joint step phase

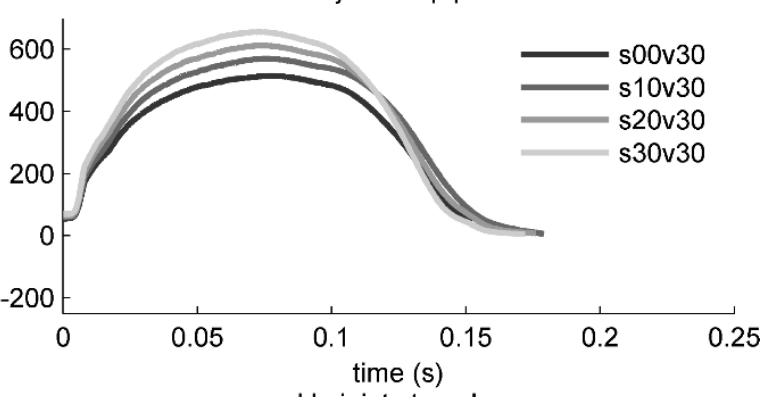

ankle joint step phase

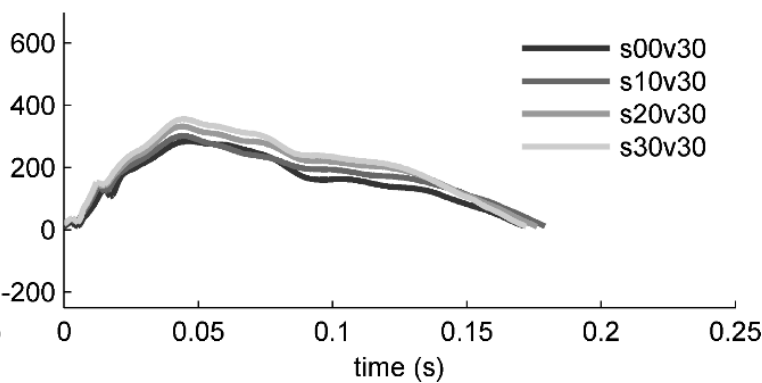

Figure 7. Example torque time histories for the joints of the stance leg in the step phase for optimisation with a) varying approach velocities and constant strength, and b) varying strengths and constant approach velocity (key: $s$ indicates strength, $v$ velocity, and the following number the increase from baseline).

In conclusion a wide range of outcomes was observed in optimisations of triple jump distance when strength and approach velocity were varied. This indicates that these two trainable factors are of great importance for triple jump performance. Across all approach velocities and strength levels either a balanced or hop-dominated technique was optimal. 


\section{CONFLICT OF INTEREST STATEMENT}

There are no conflicts of interest arising from the personal or professional associations of any of the authors.

\section{REFERENCES}

Alexander, R. McN. Optimum take-off techniques for high and long jumps. Philosophical Transactions of the Royal Society B, 1990; 329, 3-10.

Allen, S.J., King, M.A., Yeadon, M.R., 2010. Is a single or double arm technique more advantageous in triple jumping? Journal of Biomechanics, 43, 3156-3161.

Allen, S.J., King, M.A., Yeadon, M.R., 2012. Models incorporating pin joints are suitable for simulating performance but unsuitable for simulating internal loading. Journal of Biomechanics, 45, 1430-1436.

Allen, S.J., King, M.A., Yeadon, M.R., 2013. Trade-offs between horizontal and vertical velocities during triple jumping and the effect on phase distances. Journal of Biomechanics, 46, 979-983.

Allen, S.J., King, M.A., Yeadon, M.R., 2016. Optimisation of phase ratio in the triple jump using computer simulation. Human Movement Science, 46, 167-176.

Amadio, A.C., 1985. Biomechanische Analyse des Dreisprungs. Doctoral dissertation, Deutsche Sporthochschule, Koln.

Bobbert, M.F., Van Soest, A.J., 1994. Effects of muscle strengthening on vertical jump height: a simulation study. Medicine and Science in Sports and Exercise 26, 10121020.

Brimberg, J., Hurley, B., Ladany, S.P., 2006. An operations research approach to the triple jump. International Journal of Sport Management and Marketing, 1, 208-214.

Carroll, D.L., 1996. Chemical laser modeling with genetic algorithms. AIAA Journal, 34, 338-346.

Dapena, J., Chung, C.S., 1988. Vertical and radial motions of the body during the takeoff phase of high jumping. Medicine and Science in Sports and Exercise 20, 290-302.

German Athletics Federation, 2009. Biomechanical Analyses of Selected Events at the 12th IAAF World Championships in Athletics, Berlin, 15-23 August 2009. Biomechanics Report World Championships 2009 Berlin, Deutscher LeichtathletikVerband.

Hay, J.G., 1992. The biomechanics of the triple jump: a review. Journal of Sports Sciences, 10, 343-378.

Hay, J.G., 1993. Citius, altius, longius (faster, higher, longer): the biomechanics of jumping for distance. Journal of Biomechanics, 26, 7-21.

Hay, J.G., 1995. The case for a jump dominated technique in the triple jump. Track Coach, 132, 4214-4219.

Hay, J.G., 1997. Phase distances, percentages, and techniques in the men's triple jump at the 1996 U.S. Olympic trials. Track Coach, 139, 4435-4442.

Hay, J.G., 1999. Effort distribution and performance of Olympic triple jumpers. Journal of Applied Biomechanics, 15, 36-51.

King, M.A., Wilson, C., Yeadon, M.R., 2006. Evaluation of a torque-driven model of jumping for height. Journal of Applied Biomechanics, 22, 264-274.

Miller, J.A., Hay, J.G., 1986. Kinematics of a world record and other world-class performances in the triple jump. International Journal of Sports Biomechanics, 2, 272-288. 
Pain, M.T.G., Challis, J.H., 2001. The role of the heel pad and shank soft tissue during impacts: a further resolution of a paradox. Journal of Biomechanics, 34, 327-333.

Perttunen, J.O., Kyröläinen, H., Komi, P.V., Heinonen, A., 2000. Biomechanical loading in the triple jump. Journal of Sports Sciences 18, 363-370.

Ramey, M.R., Williams, K.R., 1985. Ground reaction forces in the triple jump. International Journal of Sport Biomechanics 1, 233-239.

Riener, R., Edrich, T., 1999. Identification of passive elastic joint moments in the

lower extremities. Journal of Biomechanics 32, 539-544.

Seyfarth, A., Blickhan, R., Van Leeuwen, J.L., 2000. Optimum takeoff techniques and muscle design for long jump. Journal of Experimental Biology 203, 741-750.

Simpson, S.E., Wilson, C., Kerwin, D.G., 2007. The changes in effort distribution from novice to experienced performers in the triple jump. XXV ISBS Symposium, Ouro Preto - Brazil.

Song, J.-H., Ryu, J.-K., 2011. Biomechanical analysis of the techniques and phase ratios of domestic elite triple jumpers. International Journal of Applied Sports Science, 23, 487-504.

Wilson, C., King, M.A., Yeadon, M.R., 2011. The effects of initial conditions and takeoff technique on running jumps for height and distance Journal of Biomechanics, 44, 2207-2212.

Wood, G.A., Jennings, L.S., 1979. On the use of spline functions in data smoothing. Journal of Biomechanics, 12, 477-479.

Yeadon, M.R., 1990. The simulation of aerial movement-II. A mathematical inertia model of the human body. Journal of Biomechanics, 23, 67-74.

Yu, B., Hay, J.G., 1996. Optimum phase ratio in the triple jump. Journal of Biomechanics, 29, 1283-1289.

Yu, B., 1999. Horizontal-to-vertical velocity conversion in the triple jump. Journal of Sports Sciences, 17, 221-229. 